\title{
The Method of Administration of Urea and Its Side-Effects
}

\author{
H. Fukai, R. Sato, and K. Hirata \\ The Second Department of Surgery, Niigata University School of Medicine
}

After sampling of 165 cases (465 dosis), we have established a technique of administering $30 \%$ urea solution in $6 \%$ P.V.P. intravenously with an injection speed of 100 c.c. per 20 to 30 minutes. In case of a craniotomy, the speed increased to 100 c.c. per 10 to 20 minutes so that the total dosis was finished by the time we reached the dura. As a result of the administration of the agent, we observed an obvious shrinkage of the brain in $82 \%$ of craniotomy. We observed shrinking of the dura quite frequently, when we turned the boneflap over.

Among the cases of large dosis or continuous administration, we have 74 gm per Kgm a day.

The longest was the one with 23 days of continuous administration, and in another case 50 dosis were repeated.

As to side-effects, we observed occasional vascular pain and in a prolonged continuous administration, we saw frequent thickening of vascular wall, otherwise there were no significant findings.

\section{Production and Absorption of Cerebrospinal Fluid under application of Hypotensors (Urea, PVP and Glucose) on Cerebrospinal Pressure :- Studies by Radioisotope

\author{
Kenichiro Higashi and Koji KoKura \\ The Second Surgical Division, Yamaguchi Medical College
}

Recently, attention is atracted to hypotensive action of urea and polyvinylpyrolidon (PVP).

In this study, production and absorption of cerebrospinal fluid under applications of urea, PVP and hypertonic glucose solution was investigated on unanaesthetized rabbits. Radioisotopes ( ${ }^{32} \mathbf{P}$ and RIHSA) were used for measurement of the production and absorption rate.

In those with $50 \%$ glucose solution, remarkable reduction of transition of isotope from blood to cerebrospinal fluid (production of CSF) was observed, while no such conspicuous reduction of production rate was remarked in cases with urea and PVP.

Moreover, remarkable increase of transition of isotope from cerebrospinal fluid to blood (absorption of CSF) was observed in cases with $30 \%$ urea solution. 